\title{
SAMELSON PRODUCTS OF SO(3) AND APPLICATIONS $†$
}

\author{
YASUHIKO KAMIYAMA \\ Department of Mathematics, University of the Ryukyus, Nishihara-Cho, Okinawa 903-0213, Japan \\ e-mail:kamiyama@sci.u-ryukyu.ac.jp \\ DAISUKE KISHIMOTO \\ Department of Mathematics, Kyoto University, Kyoto 606-8502, Japan \\ e-mail:kishi@math.kyoto-u.ac.jp \\ AKIRA KONO \\ Department of Mathematics, Kyoto University, Kyoto 606-8502, Japan \\ e-mail:kono@math.kyoto-u.ac.jp \\ and SHUICHI TSUKUDA \\ Department of Mathematics, University of the Ryukyus, Nishihara-Cho, Okinawa 903-0213, Japan \\ e-mail: tsukuda@math.u-ryukyu.ac.jp
}

(Received 7 November, 2006; revised 28 January, 2007; accepted 12 February, 2007)

Abstract. Certain generalized Samelson products of $S O(3)$ are calculated and applications to the homotopy of gauge groups are given.

2000 Mathematics Subject Classification. Primary 55Q15, Secondary 55P15, $54 \mathrm{C} 35$.

1. Introduction and statement of results. Each space is assumed to have the homotopy type of a CW-complex. We often make no distinction between a continuous map and its homotopy class.

Let $G$ be a topological group and let $\gamma: G \wedge G \rightarrow G$ denote the commutator of $G$. A generalized Samelson product of maps $\alpha: A \rightarrow G$ and $\beta: B \rightarrow G$ is defined as the homotopy class of the composition

$$
A \wedge B \stackrel{\alpha \wedge \beta}{\longrightarrow} G \wedge G \stackrel{\gamma}{\rightarrow} G
$$

and denoted by $\langle\alpha, \beta\rangle$. We denote the adjoint map $\Sigma A \rightarrow B G$ of a map $\alpha: A \rightarrow G$ by $\operatorname{ad}(\alpha)$. Regarding the generalized Samelson product $\langle\alpha, \beta\rangle$, Arkowitz [2] showed that

$$
\operatorname{ad}(\langle\alpha, \beta\rangle)=[\operatorname{ad}(\alpha), \operatorname{ad}(\beta)]
$$

where $[$,$] is the generalized Whitehead product.$

The purpose of this paper is to calculate certain generalized Samelson products of $S O(3)$ and to give applications to the homotopy of gauge groups. Let $\epsilon_{1}$ and $\epsilon_{3}$ be generators of $\pi_{1}(S O(3)) \cong \mathbf{Z} / 2$ and $\pi_{3}(S O(3)) \cong \mathbf{Z}$ respectively, and let $\hat{\epsilon}$ and $\iota$ be the

${ }_{\dagger}$ Supported by Grant-in-Aid for Scientific Research (B) 18340016. 
natural inclusion $\mathbf{R} P^{2} \hookrightarrow S O(3)\left(=\mathbf{R} P^{3}\right)$ and the identity of $S O(3)$ respectively. Then we shall prove the following results.

THEOREM 1.1. The order of the generalized Samelson product $\left\langle\epsilon_{3}, \hat{\epsilon}\right\rangle$ is 4 .

COROLlaRY 1.1. The order of the generalized Samelson product $\left\langle\epsilon_{3}, \iota\right\rangle$ is 12 .

Let $G$ be a compact, connected Lie group and let $P$ be a principal $G$-bundle over $S^{4}$. The gauge group $\mathscr{G}_{P}$ of $P$ is the group of all $G$-equivariant automorphisms of $P$ covering the identity of $S^{4}$. Atiyah and Bott [4] showed that

$$
B \mathscr{G}_{P} \simeq \operatorname{Map}_{P}\left(S^{4}, B G\right)
$$

where $\operatorname{Map}_{P}\left(S^{4}, B G\right)$ denotes the component of $\operatorname{Map}\left(S^{4}, B G\right)$ corresponding to the classifying map of $P$. We shall often identify $B \mathscr{G}_{P}$ with the $\operatorname{Map}_{P}\left(S^{4}, B G\right)$. For simplicity, when $G=S O(3)$ and $P$ is classified by $k \in \mathbf{Z} \cong \pi_{4}(B S O(3))$, we replace $\mathscr{G}_{P}$ by $\mathscr{G}_{k}$. Let $(n, m)$ be the GCD of $n$ and $m$. As applications of the above results, we shall prove the following results.

PROPOSITION 1.1. $\mathscr{G}_{k} \simeq \mathscr{G}_{l}$ if and only if $(12, k)=(12, l)$.

PROPOSITION 1.2.

$$
\pi_{0}\left(\mathscr{G}_{k}\right) \cong\left\{\begin{array} { l l l } 
{ \mathbf { Z } / 2 } & { k \equiv 0 ( 2 ) } \\
{ 0 } & { k \equiv 1 ( 2 ) }
\end{array} \quad \pi _ { 1 } ( \mathscr { G } _ { k } ) \cong \left\{\begin{array}{ll}
\mathbf{Z} / 2 & k \equiv 1(2) \\
\mathbf{Z} / 4 & k \equiv 2(4) \\
\mathbf{Z} / 2 \oplus \mathbf{Z} / 2 & k \equiv 0(4)
\end{array}\right.\right.
$$

REMARK 1.1. Readers may refer to [8] for the relevant results of the homotopy of $\mathscr{G}_{P}$ when $P$ is a principal $S U(2)$-bundle over $S^{4}$. Readers may also refer to [7] for an alternative calculation of $\pi_{1}\left(\mathscr{G}_{k}\right)$ in a different context.

REMARK 1.2. Regarding the homotopy of the classifying space $B \mathscr{G}_{k}$, we have the following result. Let $P$ be a principal $S U(2)$-bundle over $S^{4}$ corresponding to $k \in \mathbf{Z} \cong \pi_{4}(B S U(2))$. Since the natural projection $\mathscr{G}_{P} \rightarrow \mathscr{G}_{k}$ is a double covering, the universal covering group of the identity components of $\mathscr{G}_{P}$ and $\mathscr{G}_{k}$ are isomorphic. Then it follows from Theorem 1.5 of [10] that $B \mathscr{G}_{k} \simeq B \mathscr{G}_{l}$ if and only if $k= \pm l$.

REMARK 1.3. Let $P$ be as in Remark 1.2. Then it is straightforward to check that $\pi_{2}\left(\mathscr{G}_{k}\right) \cong \pi_{2}\left(\mathscr{G}_{P}\right)$. Hence, by a result of [8], one finds $\pi_{2}\left(\mathscr{G}_{k}\right) \cong \mathbf{Z} /(12, k)$.

2. Proofs of Theorem 1.1 and Corollary 1.1. Before starting the proofs, let us recall a result of Bott [5]. Denote a generator of $\pi_{i}(U(2))$ by $\tilde{\epsilon}_{i}$ for $i=1,3$. Then Bott [5] showed that the order of the Samelson product $\left\langle\tilde{\epsilon}_{3}, \tilde{\epsilon}_{1}\right\rangle$ is 2 and hence $\left\langle\tilde{\epsilon}_{3}, \tilde{\epsilon}_{1}\right\rangle$ is a generator of $\pi_{4}(U(2)) \cong \mathbf{Z} / 2$.

Proof of Theorem 1.1. Let $\pi: U(2) \rightarrow S O(3)$ be the natural projection. It is obvious that $\pi_{*}\left(\tilde{\epsilon}_{i}\right)=\epsilon_{i}$ for $i=1,3$. Then one has

$$
\pi_{*}\left(\left\langle\tilde{\epsilon}_{3}, \tilde{\epsilon}_{1}\right\rangle\right)=\left\langle\epsilon_{3}, \epsilon_{1}\right\rangle \in \pi_{4}(S O(3)) .
$$

Since $\pi_{*}: \pi_{4}(U(2)) \rightarrow \pi_{4}(S O(3))$ is an isomorphism, the order of $\left\langle\epsilon_{3}, \epsilon_{1}\right\rangle$ is 2 and hence $\left\langle\epsilon_{3}, \epsilon_{1}\right\rangle$ is a generator of $\pi_{4}(S O(3)) \cong \mathbf{Z} / 2$. Let $i: S^{1} \hookrightarrow \mathbf{R} P^{2}$ be the inclusion of the 
1-skeleton. Then $i^{*}(\hat{\epsilon})=\epsilon_{1}$ and, by the above observation, one can see that

$$
\left\langle\epsilon_{3}, \hat{\epsilon}\right\rangle \neq 2 \gamma
$$

for any $\gamma \in\left[S^{3} \wedge \mathbf{R} P^{2}, S O(3)\right]$.

Since $S^{3} \wedge \mathbf{R} P^{2}$ is 3-connected we have a group isomorphism

$$
\left[S^{3} \wedge \mathbf{R} P^{2}, S O(3)\right] \cong\left[S^{3} \wedge \mathbf{R} P^{2}, S p(1)\right] .
$$

By applying $\left[S^{3} \wedge \mathbf{R} P^{2},\right]$ to the fiber sequence

$$
\Omega(\operatorname{Sp}(\infty) / \operatorname{Sp}(1)) \rightarrow \operatorname{Sp}(1) \rightarrow \operatorname{Sp}(\infty) \rightarrow \operatorname{Sp}(\infty) / \operatorname{Sp}(1)
$$

we can derive an exact sequence

$$
\begin{aligned}
{\left[S^{3} \wedge \mathbf{R} P^{2}, \Omega(S p(\infty) / S p(1))\right] } & \rightarrow\left[S^{3} \wedge \mathbf{R} P^{2}, S p(\infty)\right] \\
& \rightarrow\left[S^{3} \wedge \mathbf{R} P^{2}, S p(1)\right] \rightarrow\left[S^{3} \wedge \mathbf{R} P^{2}, S p(\infty) / S p(1)\right] .
\end{aligned}
$$

Since $S^{3} \wedge \mathbf{R} P^{2}$ is 5-dimensional and $S p(\infty) / S p(1)$ is 6-connected, we obtain a group isomorphism

$$
\left[S^{3} \wedge \mathbf{R} P^{2}, S p(1)\right] \cong\left[S^{3} \wedge \mathbf{R} P^{2}, S p(\infty)\right]
$$

On the other hand, one has a sequence of group isomorphisms

$$
\left[S^{3} \wedge \mathbf{R} P^{2}, S p(\infty)\right] \cong\left[S^{4} \wedge \mathbf{R} P^{2}, B S p(\infty)\right] \cong \widetilde{K O^{0}}\left(\mathbf{R} P^{2}\right) \cong \mathbf{Z} / 4
$$

where the second and the last isomorphisms are due to Bott periodicity and a result of Adams [1], respectively. Therefore we obtain

$$
\left[S^{3} \wedge \mathbf{R} P^{2}, S O(3)\right] \cong \mathbf{Z} / 4
$$

and, by (2.1), the proof is completed.

Proof of Corollary 1.1. Since $S O(3)$ is parallelizable, the result of Atiyah [3] yields that it is stably homotopy equivalent to $\mathbf{R} P^{2} \vee S^{3}$. Then it follows from the Freudenthal suspension theorem that the cofibration $S^{3} \wedge \mathbf{R} P^{2} \stackrel{1 \wedge \hat{\epsilon}}{\longrightarrow} S^{3} \wedge S O(3) \rightarrow S^{6}$ splits as $S^{3} \wedge S O(3) \simeq\left(S^{3} \wedge \mathbf{R} P^{2}\right) \vee S^{6}$. Hence the Samelson product $\left\langle\epsilon_{3}, \iota\right\rangle$ is factored as

$$
S^{3} \wedge S O(3) \simeq\left(S^{3} \wedge \mathbf{R} P^{2}\right) \vee S^{6} \stackrel{\left\langle\epsilon_{3}, \hat{\epsilon}\right\rangle \vee \alpha}{\longrightarrow} S O(3)
$$

by a map $\alpha: S^{6} \rightarrow S O(3)$. One can see that $\alpha=\pi_{*}\left(\left\langle\tilde{\epsilon}_{3}, \tilde{\epsilon}_{3}\right\rangle\right)$, when localized at any primes but 2. It is well known that the Samelson product $\left\langle\tilde{\epsilon}_{3}, \tilde{\epsilon}_{3}\right\rangle$ is a generator of $\pi_{6}(U(2)) \cong \mathbf{Z} / 12$. Then we obtain that the order of $\alpha$ is a divisor of 12 and it is divisible by 3 , since $\pi_{*}: \pi_{6}(U(2)) \rightarrow \pi_{6}(S O(3))$ is an isomorphism. Hence, by Theorem 1.1, the order of $\left\langle\epsilon_{3}, \iota\right\rangle=\left\langle\epsilon_{3}, \hat{\epsilon}\right\rangle \vee \alpha$ is found to be 12 .

\section{Proofs of Proposition 1.1 and Proposition 1.2.}

Proof of Proposition 1.1. The idea of the proof is due to [8]. Let $e: B \mathscr{G}_{k} \simeq$ $\operatorname{Map}_{k}\left(S^{4}, B S O(3)\right) \rightarrow B S O(3)$ denote the evaluation at the basepoint of $B S O(3)$. By 
the fibration

$$
\mathscr{G}_{k} \simeq \Omega B \mathscr{G}_{k} \stackrel{\Omega e}{\longrightarrow} S O(3) \stackrel{\Gamma_{k}}{\rightarrow} \Omega_{0}^{3} S O(3),
$$

$\mathscr{G}_{k}$ can be considered as a homotopy fiber of the above map $\Gamma_{k}$. Then we shall analyze the map $\Gamma_{k}$.

By Lang [9], it is shown that the homotopy class of $\Gamma_{k}$ is $\operatorname{ad}^{3}\left(\left\langle k \epsilon_{3}, \iota\right\rangle\right)$. Since Samelson products are bilinear, we have $\Gamma_{k} \simeq k \Gamma_{1}$. By Corollary 1.1, the order of $\Gamma_{1}$ is 12. Since $\pi_{*}\left(\Omega_{0}^{3} S O(3)\right)$ is finite for all $*$, it follows from Lemma 3.2 of [6] that $\mathscr{G}_{k} \simeq \mathscr{G}_{l}$ if and only if $(12, k)=(12, l)$. Thus Proposition 1.1 is proved.

Proof of Proposition 1.2. Consider the homotopy sequence of the evaluation fibration $\Omega_{0}^{3} S O(3) \rightarrow B \mathscr{G}_{k} \stackrel{e}{\rightarrow} B S O(3)$. Then we have an exact sequence

$$
\begin{aligned}
0 & =\pi_{3}(B S O(3)) \rightarrow \pi_{2}\left(\Omega_{0}^{3} S O(3)\right) \cong \mathbf{Z} / 2 \rightarrow \pi_{2}\left(B \mathscr{G}_{k}\right) \\
& \stackrel{e_{*}}{\rightarrow} \pi_{2}(B S O(3)) \cong \mathbf{Z} / 2 \stackrel{\delta}{\rightarrow} \pi_{1}\left(\Omega_{0}^{3} S O(3)\right) \cong \mathbf{Z} / 2 \rightarrow \pi_{1}\left(B \mathscr{G}_{k}\right) \rightarrow \pi_{1}(B S O(3))=0 .
\end{aligned}
$$

Let $\Gamma_{k}: S O(3) \rightarrow \Omega_{0}^{3} S O(3)$ be as in Proposition 1.1. Then $\Gamma_{k}=\operatorname{ad}^{3}\left(\left\langle k \epsilon_{3}, \iota\right\rangle\right)$ and the connecting homomorphism $\delta$ in (3.1) is the canonical isomorphism $\pi_{2}(B S O(3)) \cong$ $\pi_{1}(S O(3))$ followed by $\left(\Gamma_{k}\right)_{*}: \pi_{1}(S O(3)) \rightarrow \pi_{1}\left(\Omega_{0}^{3} S O(3)\right)$. Hence we have

$$
\delta\left(\operatorname{ad}\left(\epsilon_{1}\right)\right)=\operatorname{ad}^{3}\left(\left\langle k \epsilon_{3}, \epsilon_{1}\right\rangle\right) .
$$

Since the order of the Samelson product $\left\langle\epsilon_{3}, \epsilon_{1}\right\rangle$ is 2, the order of its 3-fold adjoint $\operatorname{ad}^{3}\left(\left\langle\epsilon_{3}, \epsilon_{1}\right\rangle\right)$ is 2 as well. Then there exists a map $\alpha: S^{2} \rightarrow B \mathscr{G}_{k}$ satisfying the homotopy commutative diagram

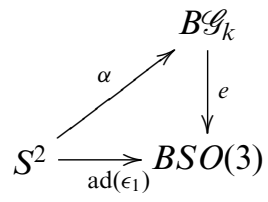

if and only if $k \equiv 0(2)$. Since $\operatorname{ad}\left(\epsilon_{1}\right)$ is the inclusion of the 2-skeleton of $B S O(3)$, $\pi_{0}\left(\mathscr{G}_{k}\right) \cong \pi_{1}\left(B \mathscr{G}_{k}\right)$ is obtained, as in the statement, by the exact sequence (3.1).

By the above argument, we have obtained $\pi_{1}\left(\mathscr{G}_{k}\right) \cong \pi_{2}\left(B \mathscr{G}_{k}\right) \cong \mathbf{Z} / 2$ if $k \equiv 1$ (2). Then we shall consider the case that $k \equiv 0$ (2) and have an exact sequence

$$
0 \rightarrow \mathbf{Z} / 2 \rightarrow \pi_{2}\left(B \mathscr{G}_{k}\right) \stackrel{e_{*}}{\rightarrow} \pi_{2}(B S O(3)) \cong \mathbf{Z} / 2 \rightarrow 0 .
$$

By Theorem 1.1, we see that the order of $\operatorname{ad}^{3}\left(\left\langle\epsilon_{3}, \hat{\epsilon}\right\rangle\right)$ is 4 . Then, quite similarly to the above, there exists a map $\hat{\alpha}: \Sigma \mathbf{R} P^{2} \rightarrow B \mathscr{G}_{k}$ satisfying the homotopy commutative diagram

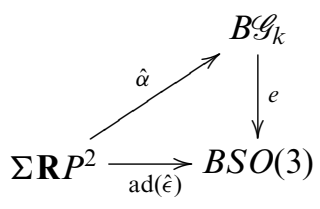


if and only if $k \equiv 0$ (4). Since $\operatorname{ad}(\hat{\epsilon})$ is the inclusion of the 3-skeleton of $B S O(3)$, we obtain, by (3.2), that $\pi_{1}\left(\mathscr{G}_{k}\right) \cong \pi_{2}\left(B_{\mathscr{G}_{k}} \cong \mathbf{Z} / 2 \oplus \mathbf{Z} / 2\right.$ when $k \equiv 0$ (4).

In the case that $k \equiv 2$ (4), we suppose that $\pi_{2}\left(B \mathscr{G}_{k}\right) \cong \mathbf{Z} / 2 \oplus \mathbf{Z} / 2$. Since $k \equiv 0$ (2), we have the above lift $\alpha: S^{2} \rightarrow B \mathscr{G}_{k}$ of $\operatorname{ad}\left(\epsilon_{1}\right)$ and it is of order 2 , by hypothesis. Note that $\Sigma \mathbf{R} P^{2}$ is the Moore space $S^{2} \cup_{2} e^{3}$ and the restriction of $\operatorname{ad}(\hat{\epsilon})$ to $S^{2}$ is $\operatorname{ad}\left(\epsilon_{1}\right)$. Then there exists the above lift $\hat{\alpha}: \Sigma \mathbf{R} P^{2} \rightarrow B \mathscr{G}_{k}$. Hence $k \equiv 0$ (4) and this is a contradiction. Therefore we have obtained that $\pi_{1}\left(\mathscr{G}_{k}\right) \cong \pi_{2}\left(B \mathscr{G}_{k}\right) \cong \mathbf{Z} / 4$ when $k \equiv 2$ (4).

\section{REFERENCES}

1. J. F. Adams, Vector fields on spheres, Ann. of Math. 75 (1962), 603-632.

2. M. Arkowitz, The generalized Whitehead product, Pacific J. Math. 12 (1962), 7-23.

3. M. F. Atiyah, Thom complexes, Proc. London Math. Soc. (3) 11 (1961), 291-310.

4. M. F. Atiyah and R. Bott, The Yang-Mills equations over Riemann surfaces, Philos. Trans. Roy. Soc. London Ser. A 308 (1983), 523-615.

5. R. Bott, A note on the Samelson product in the classical groups, Comment. Math. Helv. 34 (1960), 249-256.

6. H. Hamanaka and A. Kono, Unstable $K^{1}$-group and homotopy type of certain gauge groups, Proc. Royal Soc. Edinburgh Sect. A 136 (2006), 149-155.

7. Y. Kamiyama and D. Kishimoto, Spin structures on instanton moduli spaces, preprint.

8. A. Kono, A note on the homotopy types of certain gauge groups, Proc. Royal Soc. Edinburgh Sect. A 117 (1991), 295-297. 210.

9. G. E. Lang, The evaluation map and EHP sequences, Pacific J. Math. 44 (1973), 201-

10. S. Tsukuda, Comparing the homotopy types of the components of $\operatorname{Map}\left(S^{4}, B \operatorname{SU}(2)\right)$, J. Pure. Appl. Algebra 161 (2001), 235-243. 